\title{
A bi-criteria M-machine SDST flow shop scheduling using modified heuristic genetic algorithm
}

\author{
Ashwani Dhingra $^{1 *}$, Pankaj Chandna ${ }^{2}$ \\ ${ }^{1 *}$ Department of Mechanical Engineering, University Institute of Engineering \&Technology, Maharshi Dayanand University, Rohtak, Haryana, INDIA \\ ${ }^{2}$ Department of Mechanical Engineering, National Institute of Technology Kurukshetra,Haryana, INDIA \\ "Corresponding Author: e-mail: ashwani_dhingra1979@rediff.com,Tel+91-1262-266665, Fax. +91-1262-266665
}

\begin{abstract}
Bi-criteria flow shop scheduling problems with sequence dependent set up time (SDST) have seen an increasing attention of managers and researchers in recent years. A very restricted research has been reported on bi-criteria SDST flow shop scheduling problems dealing with due date related performance measures. In the present work, a modified heuristic based genetic algorithm (MHGA) has been developed for the aforesaid scheduling problem subject to the minimization of weighted sum of total weighted squared tardiness and makespan criterion. The modified heuristic algorithms, along with other available heuristics and dispatching rules in the literature have also being developed to solve the problem instances given by Taillard. A computational analysis has been made to evaluate the performance of the proposed MHGA for upto 200 jobs and 20 machines problems. Comparative analysis with the help of defined performance index known as relative percentage deviation (RPD) verifies that it is viable and effective approach when compared with others heuristic/dispatching rules based genetic algorithms for the SDST flow shop scheduling, especially for larger sized problems.
\end{abstract}

Keywords: Flow shop scheduling, Modified Heuristic Genetic algorithm (MHGA), Sequence dependent set up time, makespan, total weighted squared tardiness.

\section{Introduction}

Scheduling is a decision-making process that concerns the allocation of limited resources to a set of tasks with the view of optimizing one or more objectives. In today's world of global competition, effectual scheduling has become vital in order to meet customer requirements as promptly as possible while maximizing the profits. Scheduling in manufacturing systems is classically associated with scheduling a set of jobs on a set of machines in order to maximize the profit. Manufacturing system is classified as job shop, flow shop and open shop. A common job shop problem consists of $\mathrm{n}$ jobs $\left\{\mathrm{j}_{1}, \mathrm{j}_{2}, \mathrm{j}_{3}, \ldots \mathrm{j}_{\mathrm{n}}\right\}$ to be processed through $\mathrm{m}$ machine $\left\{\mathrm{m}_{1}, \mathrm{~m}_{2}, \mathrm{~m}_{3}, \ldots \mathrm{m}_{\mathrm{m}}\right\}$. Technological constraints demand that each job should be processed through the machines in a particular order and gives a significant special case named as flow shop. For a general job shop problem, the number of possible sequences are $(n !)^{m}$, where $n$ is number of jobs and $m$ is the number of machines. With the above technological constraints in case of flow shop, number of different sequences reduces to (n!). This reduced number is quite large for even temperate size problems and recognized to be NP hard problems (Garey et al., 1976; Gonzalez and Sahni, 1978; Pinedo, 2005).

Flow shop scheduling problems with sequence dependent set up time (SDST) have been one of the most renowned problems in the area of scheduling (Allahverdi, 2008; Andre's et al., 2005; Hwang et al., 1998). Sequence dependent setup times are usually found in the situation where the facility is a multipurpose machine. Some examples of SDST flow shop scheduling problem include (i) textile industry, where setup times are significant as fabric types are assigned to looms equipped with wrap chains, when the fabric type is changed on a machine, the wrap chain must be replaced and the time it takes depends on the previous and the current fabric types; (ii) the stamping plants used by most auto-makers, in such plants, sequence dependent set up time exists between manufacturing parts involves the changing of heavy dies. The case of sequence-dependent setups can be found in numerous other industrial systems also, like chemical, printing, pharmaceutical and automobile industry etc. Several researchers have given recognition to sequence dependence of setup times in the manufacturing systems. Lockett and Muhlemann (1972) 
proposed branch and bound algorithm for scheduling jobs with sequence dependent setup times on a single processor to minimize the total number of tool changes. The algorithm was computationally restrictive, suitable for only small sized problems. Corwin and Esogbue (1974) minimized makespan considering sequence dependent setup times on one of the machines subject to meeting certain due date constraints in a two machines flow shop scheduling problem. The authors concluded that the largest problem solvable on a computer was approximately 14-15 jobs. Gupta (1986) proposed a branch and bound algorithm to minimize setup cost in ' $n$ ' jobs and ' $m$ ' machines flow shop with sequence dependent setup times and concluded that this algorithm has been restricted to small problems and however heuristic rules were the preferred techniques for large size scheduling problems with computational efforts increase rapidly with problem size. Allahverdi et al. (2008) investigated the survey of flow shop scheduling problems including set up times and stated that the motivation behind SDST flow shop scheduling problem is to obtain tremendous savings when setup times are explicitly included in scheduling.

Also, most of research on SDST flow shop scheduling problems has been concentrated on single criterion problems. The most widely considered performance measures are makespan, total flow time, total tardiness, maximum tardiness and number of tardy jobs etc. Makespan and total flow time are related to maximizing system utilization while the remaining measures are related to job due dates. Flow shop scheduling with makespan criteria is very important in order to increase the productivity and maximum utilization of resources. Since, in modern manufacturing and operations management, on time delivery is a critical factor towards realizing customer satisfaction. As lack of success in meeting due dates can result in the loss of customer and market competitiveness.

Hence, scheduling problems with due date related objectives have attracted increasing attention from managers and researchers. In today's competitiveness environment, cost of production must be reduced in order to survive in this dynamic environment which has been done by effective utilisation of all the resources and production in shorter time to increase the productivity also simultaneously considering due dates of the job. As minimisation of makespan with not meeting the due date is of no use for an industry since there is loss of market competitiveness and customer.

The scheduling literature also reveals that the research on bi-criteria is mainly focused on the single-machine or two machine problems without sequence dependent set up time. Rajendran (1995) implemented a heuristic for flow shop scheduling with multiple objectives of optimizing makespan, total flow time and idle time for machines. The heuristic preference relation was proposed and used as the basis to restrict the search for possible improvement in the multiple objectives. Rahimi-Vaheda et al. (2008) considered a bi-criteria no-wait flow shop scheduling problem (FSSP) in which weighted mean completion time and weighted mean tardiness are to be minimized simultaneously with multi objective scatter search. Ravindran et al. (2005) considered flow shop scheduling with multiple objectives of makespan and total flow time together. Three heuristic HAMC1, HAMC2 and HAMC3 were proposed and concluded that proposed three heuristic yields good results than the Rajendran heuristic CR. Gupta et al. (2001) considered two machines flow shop scheduling problems with minimization of total flow time subject to the condition that the makespan of the schedule is also minimum. Gowrishankar et al. (2001) considered two types of problems, firstly $m$-machine flow shop scheduling with minimizing variance of completion times of jobs and another with minimizing sum of squares of deviations of the job completion times from a common due date. Blazewicz et al. (2005) analyzed different solution procedures for two machine flow shop scheduling problem with a common due date and weighted latework criterion. Sayin and Karabati (1999) dealt with a two machine flow shop scheduling problem for minimizing makespan and sum of completion times simultaneously. Branch and bound procedure has been developed that iteratively solves single objective scheduling problems until the set of efficient solutions has been completely enumerated. Danneberg et al. (1999) addressed the permutation flow shop scheduling problem with setup times where the jobs are partitioned into groups or families. Jobs of the same group could be processed together in a batch but the maximum number of jobs in a batch was limited and setup time depends on the group of the jobs. They proposed and compared various constructive and iterative algorithms and used the makespan and weighted sum of the completion times of the jobs as the objective function.

Toktas et al. (2004) considered the two machine flow shop scheduling by minimizing makespan and maximum earliness simultaneously. They developed a branch-and-bound procedure that generates all efficient solutions with respect to the two criteria and also proposed a heuristic procedure that generates approximate efficient solutions. Ponnambalam et al. (2004) proposed a TSPGA multi-objective algorithm for flow shop scheduling where a weighted sum of multiple objectives (i.e. minimizing makespan, mean flow time and machine idle time) was used. The proposed algorithm was evaluated by applying it to benchmark problems available in the OR-Library. Loukil et al. (2005) proposed multi-objective simulated annealing algorithm to tackle the multi-objective production scheduling problems. They considered seven possible objective functions (the mean weighted completion time, the mean weighted tardiness, the mean weighted earliness, the maximum completion time (makespan), the maximum tardiness, the maximum earliness, the number of tardy jobs). They showed that the proposed multi-objective simulated annealing algorithm was able to solve any subset of seven possible objective functions. Noorul Haq and Radha Ramanan (2006) used Artificial Neural Network (ANN) for minimizing bi-criteria of makespan and total flow time in a flow shop scheduling environment and found that performance of ANN approach is better than constructive or improvement heuristics. Rahimi-Vahed and Mirghorbani (2007) developed multi-objective particle swarm optimization for flow shop scheduling problem in order to minimize the weighted mean completion time and weighted mean tardiness simultaneously and concluded that the developed algorithm is more effective than GA for large sized problems. Dhingra and Chandna (2009) minimized multicriteria SDST flow shop scheduling including weighted sum of total tardiness, total earliness and makespan. They developed special heuristic based 
hybrid genetic algorithm in which initial feasible sequence has been obtained by special heuristic similar to NEH and optimal sequence by genetic algorithm. It has been concluded that proposed hybrid genetic algorithm showed superiority over other for 5 , 10 and 20 machines problem for said multicriteria scheduling problem. This work has been extended to include weights of the jobs and also total weighted squared tardiness criteria (Equation 2) instead of total tardiness because of the reasons discussed in section 2.2. Further proposed MHGA has been compared with total of nine available heuristics/dispatching rules based genetic algorithm for performance analysis.

Tamer Eren and Ertan Güner (2010) considered bi-criteria of minimizing a weighted sum of total completion time and total tardiness for m-identical parallel machine scheduling problem with a learning effect. Erenay et al. (2010) considered bi-criteria scheduling problem with minimization of the number of tardy jobs and average flow time on a single machine. They proposed four new heuristics (Two of these heuristics were constructive algorithms based on beam search methodology and other two were based on metaheuristics approaches using a genetic algorithm and tabu search) to solve scheduling problem. From the analysis it has been concluded that the proposed beam search heuristics produces efficient schedules and performed better than the existing heuristics. Eren (2010) minimized weighted sum of total completion time and makespan for a bi-criteria $m$-machine flow shop scheduling with sequence dependent setup times with. He developed the special heuristics and proved that the special heuristic was more effective than the others for all number of jobs and machines.

Hence, in modern manufacturing systems, for the requirement of just in time manufacturing, market competitiveness, effective utilization of resources, meeting the due dates for customer good will and satisfaction, scheduling managers has to deal with more than one interactive criterion. Therefore, bi-criteria SDST flow shop scheduling problems with due date related objective function has been considered in the present work. Further, as the problems becomes NP hard and the optimal solution is not possible in reasonable time and number of heuristic has been presented in the past but each having its own limitation as original NEH(1983) have applied to makespan problem for flow shop scheduling. Till date, various researchers have also modified NEH (Chakraborty and Laha, 2007) for other performance measures and proved to give effective results. In the present work, Modified Heuristic Genetic Algorithm (MHGA) has been proposed in which seed sequence is obtained from modified heuristic similar to NEH which considered due date and processing time simultaneously. As classical NEH considered processing times for makespan minimization and proposed heuristic also works on due date related performance measures apart from makespan for bi-criteria objective function i.e. weighted sum of total weighted squared tardiness and makespan. Initial seed sequence from other heuristic and dispatching rules available in literature have also been developed and combined with genetic algorithm for comparison with proposed MHGA. The stopping limit of all the algorithms have been considered as computational time limit which depends upon number of jobs and machines for fair comparison irrespective of number of generations as algorithm complexity of different algorithm considered to be same.

The bi-criteria scheduling problems are commonly divided into two classes. In the first class, one of the functions is considered as the objective to be optimized while the other considered as the constraints. In the second class, both the functions are weighted differently or equally and an overall objective function is defined as the weighted sum of individual functions where sum of the individual weight coefficients is unity. In the present work, the problem considered belongs to second class.

The remaining part of the paper is organized as follows. In section 2, problem description with assumptions made is presented. Section 3 describes Modified Heuristic Genetic Algorithm (MHGA). Section 4 states the analysis of experimental results \& discussions and Section 5 concludes the study with limitation of present work which further provides future work in this field.

\section{Problem Description}

In the present work, $\mathrm{n}$ job \& $m$-machine flow shop scheduling problem with sequence-dependent setup times has been considered for minimizing the weighted sum of total weighted squared tardiness and makespan. Assumptions, parameters and fitness function considered here are described below:

\subsection{Assumptions}

- All the jobs and machines are available at time Zero (i.e. the beginning of the processing horizon of sufficient time length to process all available job to completion).

- Pre-emption is not allowed. Once an operation is started on the machine it must be completed before another operation can begin on that machine.

- Machines never break down and are available throughout the scheduling period.

- All processing time on the machine are known, deterministic, finite and independent of sequence of the jobs to be processed.

- Each machine is continuously available for assignment, without significant division of the scale into shifts or days and without consideration of temporary unavailability such as breakdown or maintenance.

- The first machine is assumed to be ready whichever and whatever job is to be processed on it first.

- Each job is processed through each of the m machines once and only once. Furthermore, a job does not become available to the next machine until and unless processing on the current machine is completed i.e. splitting of jobs or job cancellation is not allowed. 
- Set up times for operations are sequence dependent and has been excluded from the processing times. It is associated with each job on each machine i.e. the time required to bring a given machine to a state, which allows the next job to commence and are immobilized to the machines.

\subsection{Parameters}

$\begin{array}{ll}j & \text { Index for Jobs } \\ i & \text { Index for Machines } \\ \alpha & \text { Weight for Total weighted squared tardiness } \\ \beta & \text { Weight for makespan } \\ C_{j} & \text { Completion time of job ' } j \text { ' } \\ d_{j} & \text { Due date of job ' } j \text { ' } \\ T_{j} & \text { Tardiness of job ' } j \text { ' } \\ w_{j} & \text { Weight for Job ' } j \text { ' }\end{array}$

$$
\begin{aligned}
& J=1,2,3 \ldots \ldots . n \\
& i=1,2,3 \ldots \ldots . m \\
& \alpha \geq 0 \\
& \beta \geq 0 \text { and } \alpha+\beta=1 \\
& J=1,2,3 \ldots \ldots . n \\
& J=1,2,3 \ldots \ldots . n \\
& J=1,2,3 \ldots \ldots . n \\
& J=1,2,3 \ldots \ldots . n
\end{aligned}
$$

2.3 Fitness Function: Fitness function considered in this work is weighted sum of total weighted squared tardiness and makespan as described below. Total weighted squared tardiness is given as (Sun et al., 1999):

$$
\begin{aligned}
& \sum_{j=1}^{n} w_{j} T_{j}^{2} \\
& T_{j}= \begin{cases}C_{j}-d_{j}, & \text { if } C_{j}-d_{j} \geq 0 \\
0, & \text { otherwise. }\end{cases}
\end{aligned}
$$

This measure of due date performance has preferred over the other due date based performance measures i.e. total weighted tardiness and maximum tardiness as in case of total weighted tardiness, a single job may contribute the majority of the tardiness without regard to how overall tardiness is being distributed and also give undesirable scenario if:

1. Tardiness characterize an important attribute to service quality; and

2. The affirmation holds that a customer's dissatisfaction tends to increase quadratically with the tardiness as proposed in Taguchi's loss function (Taguchi, 1986).

Also maximum tardiness does not distinguish tardiness that occurs in all jobs versus a single job as long as the maximum weighted tardiness is the same. Due to this, the total weighted squared tardiness is selected as one of the criteria in bi-criteria problem formulation in view of the fact that this is a compromise of the abovementioned both due date related performance measures and is a comprehensive one.

Another performance measure which is widely used for maximum utilization of resources is makespan $\left(\mathrm{C}_{\max }\right)$ for increasing productivity. Therefore, bi-criteria fitness function proposed in this work is to minimize the weighted sum of total weighted squared tardiness and makespan which is defined as:

$$
\operatorname{Min}\left[\alpha \sum_{j=1}^{n} w_{j} T_{j}^{2}+\beta C_{\max }\right]
$$

\section{Modified Heuristic Genetic Algorithm (MHGA)}

A Genetic Algorithm (GA) uses probabilistic selection as a basis for evolving a population of problem solutions. An initial population is created and subsequent generations are generated according to a pre-specified breeding and mutation methods inspired by nature. A GA must be initialized with a starting population. The methods for creating an initial population are varied: feasible only, randomized, using heuristics, etc. Classical GA generate initial population randomly and drawbacks of the algorithm is that, the choice of the initialization procedure has an important influence on the quality of solution and a better initial solution might provide better results. Due to the large search space in flow shop scheduling, it is expected that random generation of initial solutions provides relatively weak results. For this, initial solution is obtained by application of heuristics or well known dispatching rules for finding near to optimal results in a very reasonable time. In this case, the modified heuristics which is similar to NEH (Nawaz et al., 1983) is proposed for generations of initial chromosome and hybrid to GA and named as MHGA 
3.1 Outline of the MHGA: The MHGA acts as a universal search process in this algorithm. The modified heuristics (MH) developed to obtain a seed sequence based on the weighted sum of total weighted squared tardiness and makespan simultaneously. Keeping this seed sequence along with a set of (Ps - 1) random generated initial population according to population size 'Ps' since by selecting a good set of chromosomes in the initial population usually improves the performance of a genetic algorithm. The proposed MHGA is described in the following:

1. Use Modified Heuristics (MH) to generate the seed sequence in the initial population. (MH will be described in section 3.2).

2. Generation of $(\mathrm{Ps}-1)$ population randomly and combined with the seed sequence generated by MH.

3. The algorithm then creates a sequence of new populations. At each step, the algorithm uses the individuals in the current generation to create the next population. To create the new population, the algorithm performs the following steps:

a) Scores each member of the current population by computing fitness i.e. weighted sum of total weighted squared tardiness and makespan simultaneously

b) Selects members, called parents, based on their fitness.

c) Some of the individuals in the current population that have lesser fitness are chosen as elite. These elite individuals are conceded to the next population.

d) Produces offspring from the parents. Offspring's are produced either by combining the vector entries of a pair of parents - crossover or by making random changes to a single parent-mutation.

e) Replaces the current population with the children to form the next generation.

4. The algorithm stops when one of the stopping criteria is met.

3.2 Proposed Modified Heuristic (MH): A modified heuristic based GA is developed for solving jobs scheduling problem in $n \times m$ flow shops with due date constraints including sequence dependent set up times. Proposed MH is described as below:

\section{Step 1}

Arrange the jobs in ascending order of the sum of processing times of each job on all the machines and due date by weight of each job (i.e. $\left\{\left(\sum_{i=1}^{m} P_{i j}+d_{j}\right) / w_{j}\right\}$ ).

Step 2

Set $k=2$. Pick the first two jobs from the rearranged jobs list and schedule them in order to minimize the weighted sum of total weighted squared tardiness and makespan as if there are only two jobs. Set the better one as the current solution.

Step 3

Increment $k$ by 1 . Generate $k$ candidate sequences by inserting the first job in the remaining job list into each slot of the current solution. Amongst these Candidates, select the best one with the least partial minimization of the weighted sum of total weighted squared tardiness and makespan. Update the selected partial solution as the new current solution.

Step 4

If $k=n$, a schedule (the current solution) has been found and stop. Otherwise, go to step 3 .

\section{Results and Discussions}

In this work, all the experimental tests are conducted on a personal computer with P IV/2 with 1 GB Ram. In MHGA, modified heuristic has been proposed for generating the initial chromosome followed by genetic algorithm for bi-criteria SDST flow shop scheduling problems in MATLAB environment with the objective of weighted sum of total weighted squared tardiness and makespan. The evaluation of all the algorithms implemented has been carried out using the instances (DD_SDST_10) developed by Taillard (1993) upto 200 jobs and 20 machines under SDST environment with due dates and weights allotted to each job. As proposed GA generate initially, Ps-1 random population and with a sequence preset by modified heuristic or any other heuristics/ dispatching rule, so for each instance, we have run GA five times for taking final average. Also for reasonable comparison, termination of GA has been fixed to computational time limit based criteria, which is $n \times m \times 0.25 \mathrm{sec}$ onds which allows more time to run as the size of problem increases. Initial chromosome is obtained by the modified heuristic as explained in section 3.2. Apart from $\mathrm{MH}$, various other heuristics and dispatching rules as shown in Table 1 have also been developed for comparative analysis of proposed MHGA for bi-criteria SDST flow shop scheduling problem.

Also initial sequence obtained from the various heuristics/dispatching rule as stated in Table 1 is used as a seed sequence along with a set of other population for finding near to optimal solution by genetic algorithm. Therefore, various heuristic/dispatching rule based genetic algorithms are also developed namely NEH(GA), EDD(GA), SPT(GA), EWDD1(GA), EWDD2(GA), EWDD1_NEH(GA), EWDD2_NEH(GA) RANDOM(GA) for comparative analysis with MHGA. Parameters fixed for genetic algorithm is given in Table 2 .

The most common performance measure to compare all the algorithms is the relative percentage deviation $(R P D)$ which is computed as (Naderi et. al., 2009): 


$$
\text { Relative percentage deviation }(R P D)=\frac{\text { Method }_{\text {sol }}-\text { Best }_{\text {sol }}}{B e s t_{\text {Sol }}} \times 100
$$

Where Method $_{\text {sol }}$ is the solution obtained by a given method and Best $t_{s o l}$ is the best solution obtained among all the methods or the best known solution. RPD nearer to zero gives the best results. Best ${ }_{\text {sol }}$ can be found among the results obtained by running GA five times for a particular problem and method solution is final average solution given by the algorithm for all the five runs. Comparison of RPD ( Equation 3) of all the heuristics/dispatching rules based genetic algorithm for three set of weights ( i.e. $(0.75,0.25),(0.5,0.5)$ and $(0.25,0.75))$ for the defined fitness function are shown in Figure 1, Figure 2 and Figure 3.

Table 1. Different heuristics/dispatching rules

\begin{tabular}{|c|c|c|c|}
\hline S.No & $\begin{array}{l}\text { Dispatching Rule } \\
\text { /Heuristic }\end{array}$ & Description & Reference \\
\hline 1. & $\begin{array}{lll}\begin{array}{l}\text { Shortest } \\
\text { (SPT) }\end{array} & \text { processing } & \text { time } \\
\end{array}$ & $\begin{array}{l}\text { Initially jobs are processed according to sum of } \\
\text { each job on all the machines in ascending order }\end{array}$ & $\begin{array}{l}\text { Panwalker and Iskander } \\
\text { (1977) }\end{array}$ \\
\hline 2. & Earliest due date (EDD) & Jobs are processed according to earliest due dates. & Kim (1993) \\
\hline 3. & $\begin{array}{l}\text { Earliest weighted due date rule } \\
\text { (EWDD1) }\end{array}$ & Arrange the jobs according to least value of $\frac{d_{j}}{w_{j}}$. & $\begin{array}{l}\text { Parthasarathy and } \\
\text { Rajendran (1997) }\end{array}$ \\
\hline 4. & $\begin{array}{l}\text { Earliest weighted due date } \\
\text { (EWDD2) }\end{array}$ & $\begin{array}{l}\text { Initially arrange the jobs according to least value } \\
\text { of } w_{j} d_{j} \text {. }\end{array}$ & Vallada et al. (2008) \\
\hline 5. & Original NEH & $\begin{array}{l}\text { Schedule the jobs initially in descending order } \\
\text { of } \sum_{i=1}^{m} P_{i j} \text {. }\end{array}$ & Nawaz et al. (1983) \\
\hline 6. & Random & $\begin{array}{l}\text { Randomly generation of population according to } \\
\text { population size as in classical genetic Algorithm }\end{array}$ & Nazif and Lee (2009) \\
\hline 7. & EWDD1_NEH & $\begin{array}{l}\text { Initially jobs are ordered according to least value of } \\
\frac{d_{j}}{w_{j}} \text { and then final sequence is obtained by NEH } \\
\text { procedure }\end{array}$ & $\begin{array}{l}\text { Ruiz and Stutzle, } \\
\text { (2008) }\end{array}$ \\
\hline 8. & NEH_EDD & $\begin{array}{l}\text { Initially jobs are arranged according to earliest due } \\
\text { date and then final sequence of jobs is obtained by } \\
\text { NEH procedure }\end{array}$ & $\begin{array}{l}\text { Holthaus and } \\
\text { Rajendran (2005). }\end{array}$ \\
\hline 9. & EWDD2_NEH & $\begin{array}{l}\text { Initially jobs are ordered according to least value of } \\
d_{j} w_{j} \text { and then final sequence is obtained by NEH } \\
\text { procedure. }\end{array}$ & $\begin{array}{l}\text { Dhingra \& Chandna } \\
(2010)\end{array}$ \\
\hline
\end{tabular}

Table 2. Parameters for Genetic Algorithm

\begin{tabular}{cc}
\hline \hline Parameter & Value \\
\hline Population size & 50 \\
Elite Count & 2 \\
Crossover fraction & 0.8 \\
Mutation fraction & 0.15 \\
Stopping Criteria & $\mathrm{n} \times \mathrm{m} \times 0.25$ \\
(Time limit in seconds) & \\
Migration rate & 0.2 \\
Migration Interval & 20 \\
Crossover function & Order \\
Mutation function & Reciprocal \\
& Exchange \\
\hline \hline
\end{tabular}




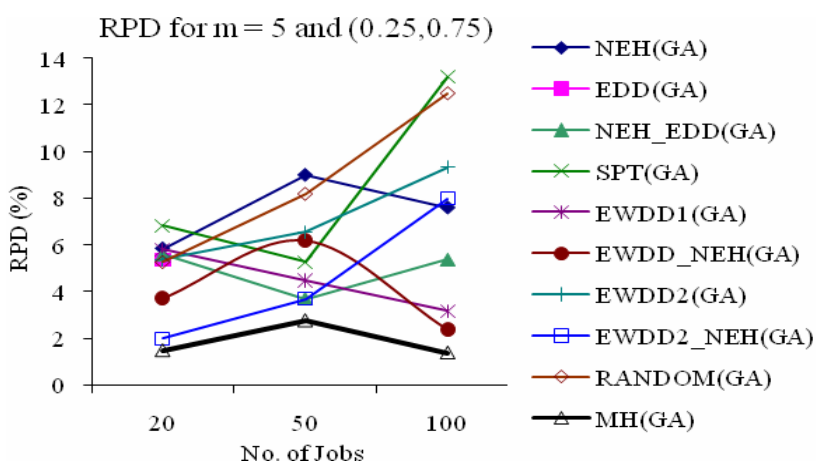

(a)

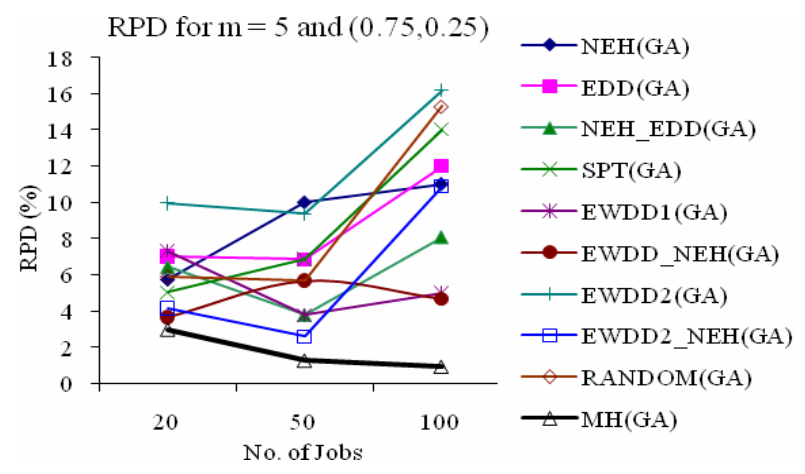

(b)

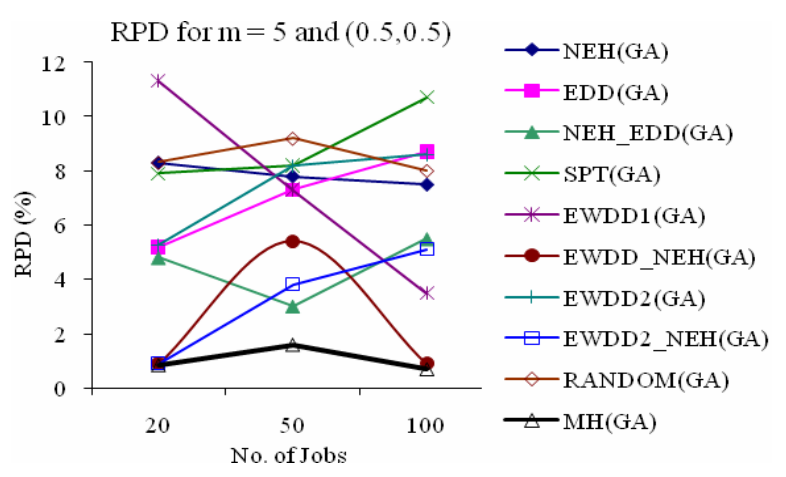

(c)

Figure 1. RPD for five machines problems for $(\alpha, \beta)=$ (a) $(0.25,0.75)$ (b) $(0.75,0.25)$ (c) $(0.5,0.5)$

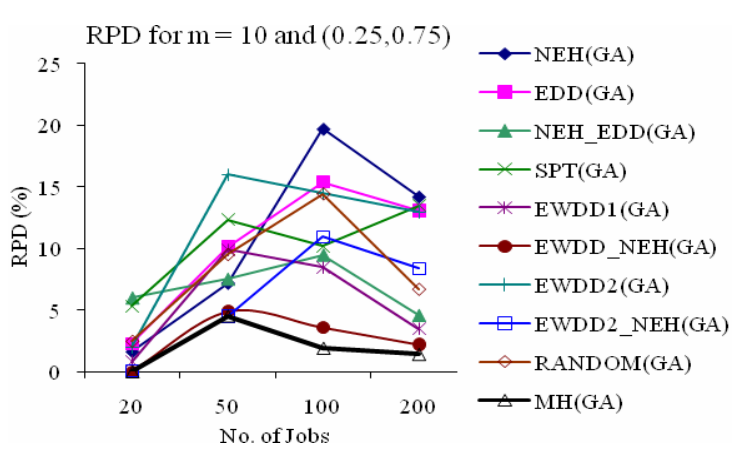

(a)

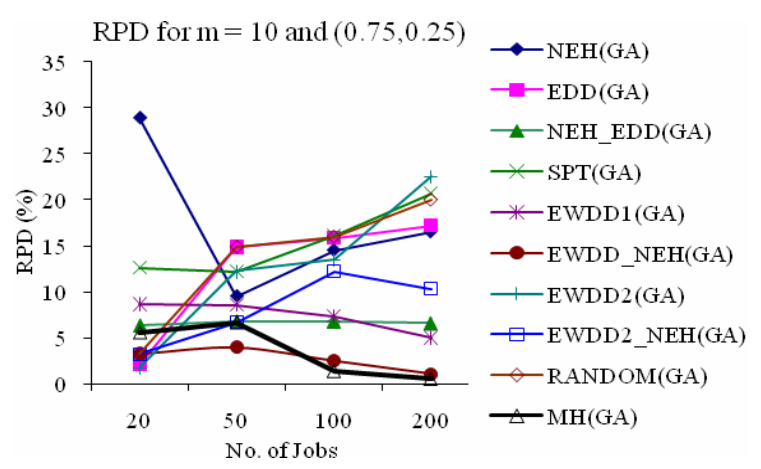

(b)

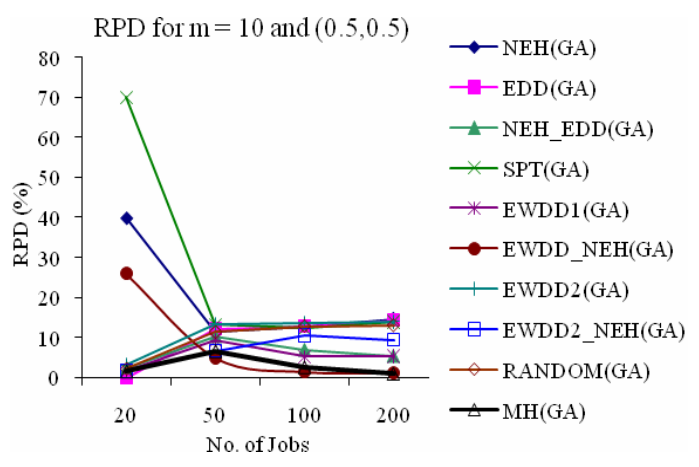

(c)

Figure 2. RPD for ten machines problems for $(\alpha, \beta)=$ (a) $(0.25,0.75)$ (b) $(0.75,0.25)$ (c) $(0.5,0.5)$ 


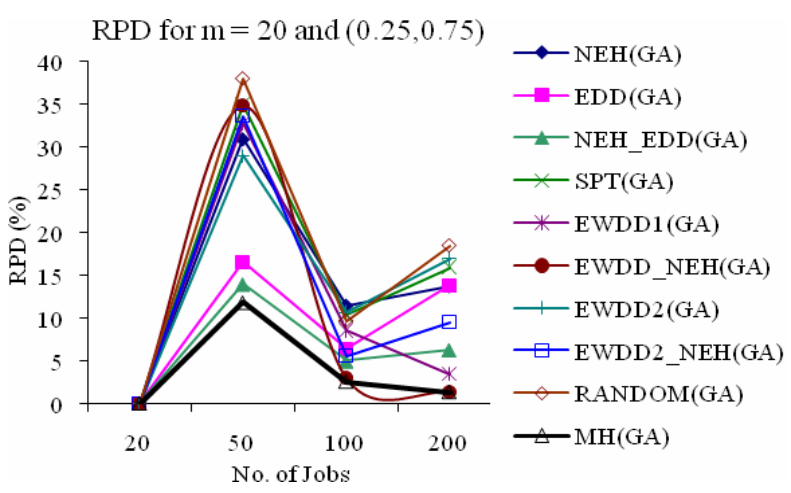

(a)

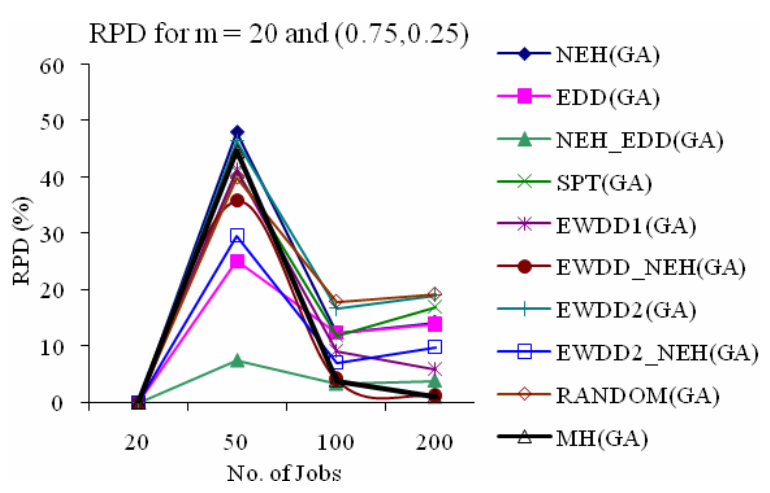

(b)

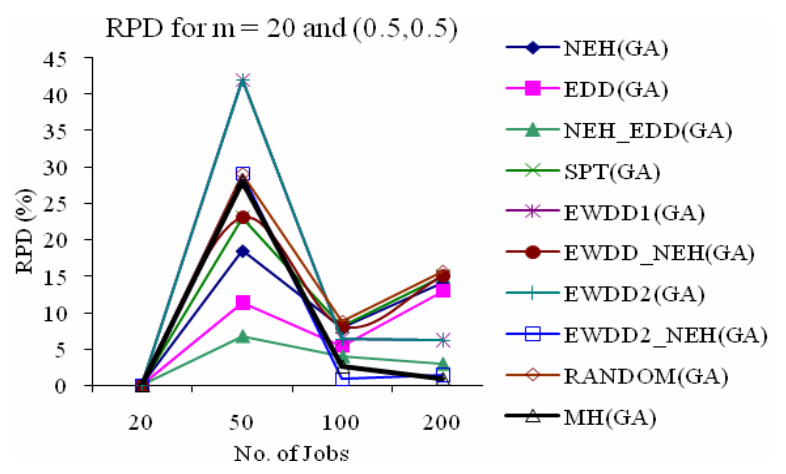

(c)

Figure 3. RPD for twenty machines problems for $(\alpha, \beta)=\mathbf{( a )}(0.25,0.75) \mathbf{( b )}(0.75,0.25) \mathbf{( c )}(0.5,0.5)$

As shown in Figure 1 upto five machines problems, the performance of proposed MHGA gives better results than others for all the three sets of weights considered with mean RPD of $1.75 \%, 1.05$ and $1.9 \%$ respectively. However, for ten machines problem, initially EWDD1_NEH (GA) performs well for weight values of $(0.75,0.25)$ and $(0.5,0.5)$ but restricted to small number of jobs as shown in Figure 2 (a) and (b). As number of jobs increases, performance of MHGA also increases well and mean RPD for ten machines problem obtained is $3.3 \%, 3.15 \%$ and $1.95 \%$ for all the three sets of weights. As shown in Figure 3 for twenty machine problems, almost all the algorithms produce comparable results (especially for 20 jobs) and as number of jobs increases, performance of MHGA again increases with mean RPD obtained is $12.4 \%, 7.9 \%$ and $4 \%$ respectively for all the three sets of weights.

Hence, upto five machine problems, MHGA proves to be very effective in all the cases with any number of jobs. However, as the number of machines increased (especially for 20 jobs) almost all the algorithm gives comparable results. But as number of jobs increases, performance of MHGA improves. EWDD1 NEH (GA) and NEH EDD (GA) also performed well in some cases as stated in literature but restricted to small number of jobs as shown. Overall, proposed MHGA shows superiority especially for large sized SDST flow shop scheduling problems with bi-criteria of weighted sum of total weighted squared tardiness and makespan.

\section{Conclusion}

In the present work, SDST flow shop scheduling problem have been considered with minimizing the bi-criteria of weighted sum of total weighted squared tardiness and makespan. Modified heuristic based genetic algorithm (MHGA) has been proposed and compared with several other heuristic/dispatching rules based genetic algorithms. All the GA's have been tested on instances derived by Taillard (1993) upto 200 jobs and 20 machines for all the three weights $(\alpha, \beta)$ for the defined fitness function (i.e. $(0.75$, $0.25),(0.5,0.5) \&(0.25,0.75))$. EWDD1_NEH (GA) and NEH_EDD (GA) performed well in some cases as reported in literature but limited to smaller problem size. However, as problem size increases MHGA proves to be effective under SDST environment. Hence, it may be concluded that the performance of the MHGA when compared to other heuristics/dispatching rules based genetic algorithms discovered the absolute superiority of proposed MHGA for large size SDST flow shop scheduling problem with considered bi-criteria fitness function. In the present work, MHGA for a defined bi-criteria performance measures has been considered and work can be extended to other multi-criteria fitness function. Designing optimal parameters using design of 
experiments approach for MHGA can also be the scope for the further analysis for different size SDST flow shop for achieving accurate results for multi-criteria fitness function.

\section{Acknowledgments}

The authors would like to thank the anonymous reviewers for their valuable suggestions in revising the manuscript.

\section{References}

Allahverdi A., Ng C.T., Cheng T.C.E. and Kovalyov M.Y., 2008. A survey of scheduling problems with setup times or costs, European Journal of Operation Research, Vol. 187, No.3, Vol.985-1032.

Andre's C., Albarracn J. M., Tormo G., Vicens E. and Garcia Sabater J.P., 2005. Group technology in a hybrid flow shop environment: A case study, European Journal of Operation Research, Vol.167, pp.272-281.

Blazewicz J., Pesch E., Sterna M. and Werner F., 2005. A comparison of solution procedures for two-machine flow shop scheduling with late work criterion, Computer and Industrial Engineering, Vol. 49, pp.611-624.

Chakraborty U.K. and Laha, D., 2007. An improved heuristic for permutation flowshop scheduling, International Journal of Information and Communication Technology, Vol. 1, No.1, pp. 89 - 97.

Corwin B. D. and Esogbue A. O., 1974. Two machine flow shop scheduling problems with sequence dependent setup times: a dynamic programming approach, Naval Research Logistics Quarterly, Vol. 21, pp. 515-523.

Danneberg D., Tautenhahn T and Werner F., 1999. A comparison of heuristic algorithms for flow shop scheduling problems with setup times and limited batch size, Mathematical and Computer Modeling, Vol. 29, pp.101-126.

Erenay F.S. , Sabuncuoglu I., Toptal A. and Tiwari M.K ., 2010. New solution methods for single machine bi-criteria scheduling problem: Minimization of average flow time and number of tardy jobs, European Journal of Operational Research, Vol.201, No.1, pp. $89-98$.

Dhingra A. and Chandna P., 2009. Hybrid genetic algorithm for multicriteria scheduling with sequence dependent set up time, International Journal of Engineering, Vol. 3, No. 5, Vol. 510 - 520.

Dhingra A. and Chandna P., 2010. Hybrid genetic algorithm for SDST flow shop scheduling with due dates: a case study, International Journal of Advanced Operation Management (In Press).

Garey M.R., Jonson D.S. and Sethi, R.,1976. The complexity of flow shop and job shop scheduling, Mathematics of Operation Research, Vol. 1, pp.117-129.

Gonzalez T. and Sahni S., 1978. Flow shop and job shop scheduling: complexity and approximation, Operation Research, Vol. 26, pp.36-52.

Gowrisankar K., Chandrasekharan R. and Srinivasan G., 2001. Flow shop scheduling algorithm for minimizing the completion time variance and the sum of squares of completion time deviations from a common due date, European Journal of Operation Research', Vol. 132, pp. 643-665.

Gupta S.K., 1986. N jobs and m machines job-shop problems with sequence-dependent set-up times, International Journal of Production Research, Vol. 20, No.5, pp. 643-656.

Holthaus O and Rajendran C., 2005. A fast ant-colony algorithm for single-machine scheduling to minimize the sum of weighted tardiness, The Journal of the Operational Research Society, Vol. 56, No. 8, pp. 947-953

Hwang H. and Sun J. U., 1998. Production sequencing problem with reentrant workflows and sequence dependent setup times, International Journal of Production Research, Vol. 36, pp.2435-2450.

Jatinder N.D., Venkata G., Neppalli R. and Werner F., 2001. Minimizing total flow time in a two-machine flow shop problem with minimum makespan, International Journal of Production Economics, Vol. 69, pp. 323-338.

Kim Y.D., 1993. Heuristics for flow shop scheduling problems minimizing mean tardiness, Journal of Operational Research Society, Vol. 44, pp.19-28.

Lockett A. G. and Muhlemann A. P., 1972. Technical notes: a scheduling problem involving sequence dependent changeover times, Operation Research, Vol. 20, pp. 895-902.

Loukil T., Teghem J. and Tuyttens D., 2005. Solving multi-objective production scheduling problems using metaheuristics, European Journal of Operation Research, Vol. 161, pp. 42-61.

Naderi B., Zandieh M. and Roshanaei V., 2009, Scheduling hybrid flow shops with sequence dependent setup times to minimize makespan and maximum tardiness, International Journal of Advanced Manufacturing Technology, Vol. 41, pp.1186-1198.

Nawaz M., Enscore E. and Ham I., 1983. A heuristic algorithm for the m-machine n-job flow shop sequencing problem ,Omega, Vol.11, Vol. 91-95.

Nazif H. and Lee L.S., 2009. A genetic algorithm on single machine scheduling problem to minimize total weighted completion time, European Journal of Scientific Research, Vol.35, No.3, pp.444-452.

Noorul Haq and Radha Ramanan T., 2006. A bi-criterion flow shops scheduling using artificial neural network, International Journal of Advanced Manufacturing Technology, Vol. 30, pp. 1132-1138.

Parathasarthy S and Rajendran C., 1997. A simulated annealing heuristic for scheduling to minimize mean weighted tardiness in a flow shop with sequence dependent setup times of jobs-a case study, Production Planning and Control, Vol. 8 ,pp.475-483. 
Panwalker $S$ and Iskander $W$., 1977. A survey of scheduling rules, Operation Research, Vol. 25, pp. 45-61.

Pinedo M., 2005.Scheduling theory, algorithms, and system, (Prentice Hall)

Ponnambalam S. G., Jagannathan H., Kataria M. and Gadicherla A., 2004. A TSP-GA multi-objective algorithm for flow shop scheduling, International Journal of Advanced Manufacturing Technology, Vol. 23, pp. 909-915.

Rajendran C., 1995.Theory and methodology heuristics for scheduling in flow shop with multiple objectives, European Journal of Operation Research, Vol. 82, pp.540 -555.

Rahimi-Vahed A.R., Javadi B., Rabbani M. and Tavakkoli-Moghaddam R., 2008.A multi-objective scatter search for a bi-criteria no-wait flow shop scheduling problem, Engineering Optimization, Vol. 40, No.4, pp. 331 -346.

Rahimi-Vahed R., Mirghorbani S. M., 2007. A multi-objective particle swarm for a flow shop scheduling problem, Combinatorial Optimization, Vol. 13, pp.79-102.

Ravindran D., Noorul Haq A., Selvakuar S.J. and Sivaraman R., 2005. Flow shop scheduling with multiple objective of minimizing makespan and total flow time, International Journal of Advanced Manufacturing Technology, Vol. 25, pp.10071012.

Ruiz R and Stutzle T., 2008. An iterated greedy heuristic for the sequence dependent setup times flowshop problem with makespan and weighted tardiness objectives, European Journal of Operational Research, Vol.187, pp.1143-1159.

Sayin S. and Karabati S., 1999. A bi-criteria approach to the two-machine flow shop scheduling problem, European Journal of Operation Research, Vol. 113, pp. 435-449.

Sun X., Noble J.S. and Klein C.M., 1999. Single-machine scheduling with sequence dependent setup to minimize total weighted squared tardiness, IIE Transactions, Vol. 31, pp.113 - 124.

Taguchi G., 1986. Introduction to Quality Engineering. (Asian Productivity organization), Tokyo, Japan.

Taillard E.,1993. Benchmarks of basic scheduling problems, European Journal of Operation Research, Vol. 64, pp.278-285.

Toktas B., Azizoglu M. and Koksalan S.K., 2004. Two-machine flow shop scheduling with two criteria: Maximum earliness and makespan, European Journal of Operational Research, Vol. 157, pp. 286-295.

Eren, T., 2010. A bicriteria M-machine flow shop scheduling with sequence-dependent setup times, Applied Mathematical Modelling, Vol. 34, No.2, pp. 284 - 293.

Tamer Eren and Ertan Guner, 2009. A bi-criteria parallel machine scheduling with a learning effect, International Journal of Advanced Manufacturing Technology, Vol.40, pp.1202-1205.

Vallada E., Ruiz R. \& Minella G., 2008. Minimizing total tardiness in the $m$-machine flowshop problem: A review and evaluation of heuristics and metaheuristics, Computers and Operations Research, Vol. 35, No. 4, pp. 1350-1373.

\section{Biographical notes}

Ashwani Dhingra is serving in Department of Mechanical Engineering, University Institute of Engineering and Technology at Maharshi Dayanand University Rohtak. He has obtained Bachelors degree from Maharshi Dayanand University Rohtak Haryana India in 2002, Masters Degree from Panjab Engineering College Chandigarh India and submitted doctorate degree thesis in Mechanical Engineering from National Institute of Technology (NIT), Kurukshetra, Haryana, India. His areas of interest include Metaheuristics, Combinatorial optimization, Operations research, Lean manufacturing, Sequencing and Scheduling etc. He is also reviewer of few journals of repute. He has published a number of research articles in international/national journals and conferences.

Pankaj Chandna is serving as an Associate Professor in the Department of Mechanical Engineering at NIT, Kurukshetra, Haryana, India. After being graduated in Mechanical Engineering from NIT kurukshetra (formerly REC kurukshetra) in 1989 and received his Ph.D degree in 2003 from Kurukshetra University, Kurukshetra. His current research areas of interest include sequencing and scheduling, ergonomics, anthropometry, inventory management and quality control etc. He has more than thirty five research papers in international/national journals and conferences to his credit.

Received May 2010

Accepted May 2010

Final acceptance in revised form August 2010 